\title{
Decision Making in Networks: An Experiment on Structure Effects in a Group Dictator Game
}

\author{
Bernhard Kittel $^{*} \quad$ Wolfgang J. Luhan ${ }^{\dagger}$
}

\begin{abstract}
In this study we examine the impact of communication networks on decision making in five-person committees. Within the framework of a group dictator game the committees must reach a majority choice for one collective transfer. We focus on the effect of the group structure on the collective decisions, and explore the effect of incomplete and asymmetric information on voting outcomes by defining three different networks structures: the unconnected network, a circle and a star. As expected the connections improved coordination within the committees, however, we find no significant difference between the network forms concerning the transfers chosen.
\end{abstract}

Keywords: experiment, network, dictator game, team behavior, communication effects.

JEL Classification Codes: C72, C92, D70, D71.

Published as: Kittel, B. and W.J. Luhan (2013), 'Decision Making in Networks: An Experiment on Structure Effects in a Group Dictator Game', Social Choice and Welfare, 40, 141-154.

*Institut für Wirtschaftssoziologie, Universität Wien, Austria, e-mail: bernhard.kittel@univie.ac.at

${ }^{\dagger}$ Department of Economics, Ruhr-Universität Bochum, Germany; e-mail: wolfgang.luhan@rub.de. 


\section{Introduction}

Collective decisions are often made under the condition of incomplete and asymmetric information among group members. For example, a committee may be chaired by a person that has privileged access to information or a committee may consist of individuals who trust some but not all of the other committee members and hence share information only with a subset of the members. In fact, any organization that relies on committees to decide on a policy is to some extent confronted with an asymmetric distribution of information across agents with heterogeneous individual preferences.

Such settings can be considered abstract versions of decision processes repeatedly observed in policy networks such as the European Union. The European Commission, for example, develops directives in close cooperation with member states and various lobby organizations that often do not - or only very restrictively - share information among each other (Thomson et al. 2006; Thurner and Binder 2008). Another relevant setting is a situation in which non-governmental organizations (NGOs) are involved in a policy process. These might on the one hand, as above, be considered as one 'composite' agent by the other agents involved in the decision making process. The NGOs might, on the other hand, actually base their decisions about their joint positions within a larger actor constellation on information shared with a subset of friendly NGOs but not with others. A growing body of empirical studies of NGO influence on EU policy-making is suggestive of the difficulties involved in coordination in such situations (Biliouri 1999; Beyres 2004; Greenwood 2003; Hallstrom 2004; Mahoney 2004). Similar real-world situations can also be observed in more mundane settings such as committee decisions in local municipalities, associations, or universities.

In this study, we abstract from the particularities of such situations and aim to single out some of the mechanisms of decision making in a committee. The focus of our analysis lies on the effects of communication structures within a group on the outcome of the collective decision. Possible information asymmetries may generate uncertainty about other group members' behavior and thereby induce power asymmetries within a group.

Our main research question is which impact different network constellations - implying different distributions of information - have on the probability of articulation majority decision and on the content of the decision. We explore the effect of incomplete and asymmetric information on majority formation by defining two different networks structures, a circle and a star (see, e.g., Bala and Goyal 2000) and 
compare these to an unconnected network without communication as a reference treatment.

In recent years, there has been an upswing in network research (for excellent surveys see Dutta and Jackson 2003, Jackson and Watts 1998, Jackson and Wolinsky 1996, Kosfeld 2004, Goyal 2007). The literature can be roughly divided in two strands: the first examining the formation of networks, the second dealing with the impact on outcomes. Our research question is related to this second strand where network structures are exogenously specified and the impact on theoretical predictions and empirical results is observed (see, e.g. Bala and Goyal 2000, Glaeser et al. 1996, Morris 2000, Charness et al. 2007, Chwe 2000). We do not endogenize network formation or the shape of emerging networks.

In order to control for distributional concerns within the group, we restrict our analysis to collective problem solving (in contrast to spot contracts, distributive bargaining, and positive coordination; see Scharpf 1997). We frame the situation as a dictator game. The problem to be solved in this game is to agree on an amount to be transferred from all committee members to a receiver group of equal size. The responsibility for a dependent party without entitlement appears to reflect the reality of political decision making in the context of problem solving better than the allocation or reallocation of endowments within a group of decision makers. Since the decision does not affect within-group redistribution but spreads the costs equally among the committee members we can disregard behavior related to such redistributional conflict (see, e.g., Frohlich and Oppenheimer 2007, Tyran and Sausgruber 2006, Traub et al. 2009).

The standard game theoretic prediction, assuming fully informed rational agents maximizing their own profits, is the smallest possible transfer. Therefore the network structure should not have any effect on the outcome and the size of the transfer chosen should be zero in all instances. When relaxing these theoretical assumptions, a coordination problem arises where the communication structure might play a decisive role.

Thus the focus is on the extent to which individual revenue maximization and social preferences, captured by the size of the transfer, are represented in the group decision. Real-world counterparts would be situations in which money is spent collectively without clear positive effects (apart from intrinsic motivations) on the committee members themselves, while others will profit from the transfer.

The paper is structured as follows: In the second section, the experimental design is outlined. The third section explains the analytical framework, the theoretical argument and the hypotheses. Section four 
presents details of the experimental setup. In section five, we report our findings and section six concludes.

\section{Experimental Design}

We study the impact of communication networks on group decisions in a highly controlled laboratory environment. We form groups of five anonymous subjects with the task to allocate a pie between themselves and a second group of five anonymous subjects that is actually present in the lab, but as anonymous as the members of the dictator group. The recipients group has no other option than to take whatever the dictator allocates to them, with every transfer being distributed equally amongst the group members. If a majority (at least three out of five group members) chooses a certain allocation, it is implemented, whereas in case of no majority choice the dictator group earns nothing and the payoff for each member of the recipient group is a random draw from a uniform distribution covering the range of possible transfers.

Our examination of the communication structure differs significantly from previous studies on the effects of communication. In the realm of simple bargaining games the research on communication effects largely concentrated on the communication media (Roth 1995; Sia, Tan and Wei 2002; Brosig et al. 2003; Luhan et al. 2009). A considerable, yet growing, body of experimental literature has examined the equilibrium choice in coordination games with a particular focus on the impact of communication and learning in repeated interaction (see Ochs 1995, for an overview).

We implemented the decision task as a dictator game rather than a standard coordination game. In this game we expect social preferences to generate intragroup conflicts, while featuring obvious focal points for coordinating collective action as is described in the next section.

In order to test the influence of communication networks on the group decision making process, we first designed a baseline treatment with an unconnected group (nocom). In this treatment the members of the dictator group individually set their transfers without any means of coordination. If no majority choice is reached in the first attempt, the group members may choose transfers for a second time without any information on the previous choices of the other group members.

But the setting of an unconnected network as a decision making group is most unrealistic and serves only as a reference point. Communication channels of various forms can be found in any political context, either structured or unstructured. As we try to examine the basic effects of communication networks we want to exclude any effects of the actual communication channels used and restrict communication 
Figure 1: Star-shaped network

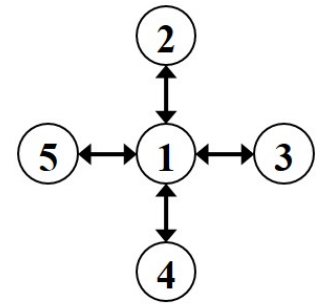

Figure 2: Circular network

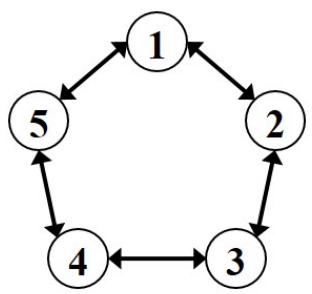

to a very limited and strictly structured form.

We implemented this idea as a two stage game, where in the first stage the members of the dictator group individually propose a share of their endowment to be transferred to the other group. This information is then revealed selectively to other group members according to the network structure and thus serves as an indicator of the transfer that the respective subject is willing to choose. In the second stage, individuals simultaneously make a decision on the share to be transferred. Only if a majority of the group enters the same amount in this stage, the transfer is effectuated for all members of the group. Thus, the individual share proposed by a majority becomes the group share. As in the nocom treatment the group had a second chance to form a majority, should they have failed to do so in the first attempt. They would go through both stages again, submitting a proposal in the first stage, which would be revealed, and subsequently choosing the transfer.

We model two network structures, a star-shaped network with one central group member (star) - as depicted in Figure 1 - and a circular network (circle) - as depicted in Figure 2. The network structures are common knowledge to the group members.

In the star-shaped network, only the central member (member 1) can see all proposals and only her proposal is visible to all other group members. In the circular network, all agent are informed about the proposals of their immediate neighbors to the right and to the left. We discuss the hypothesized implications of these two network structures in the next section.

\section{$3 \quad$ Theory and Hypotheses}

The dictator game was first introduced by Kahneman et al. (1986) in an empirical study on fairness in the market place. We know from an extensive experimental literature that, typically, individuals show social preferences to a certain, though individually varying, extent 
(see, e.g., Blount 1995; Fehr and Schmidt 1999; Bolton and Ockenfels 2000). Camerer (2003) reports average transfers in the range from 10 to $23 \%$ in several individual dictator games. In the context of the extensive literature on individual behavior we thus ask whether equityand reciprocity-oriented individuals change their behavior in a dictator game when they are part of a group.

The common notion of a fair split might serve as a clue in the process of majority formation. This notion was repeatedly observed in experimental studies, for example on the ultimatum game, where general transfers exceed $30 \%$ of the pie (e.g. Camerer and Thaler 1995) with the equal split being the modal offer. Recipients repeatedly reject transfers of less than 20\% in such situations (Roth 1995; Pillutla and Murnighan 1995).

As mentioned above, the actual task is only a framework for the group decision-making process and its final outcomes, which is the focus of our study. In the decision-making group every member $(n=$ 5 ) has to decide individually on a transfer $x$ of "her share" of the stake, $c=C / n$, with $x \leq c$. If a majority of at least three group members individually choose the same transfer $x$, this is set to be every group member's transfer and $X=n x$ is transferred to the recipients group. Irrespective of the individual choices, $x$ is deducted from each member's initial endowment, thereby creating payoffs of

$$
y_{d}=c-x
$$

for the members of the dictator group and

$$
y_{r}=x
$$

for each member of the recipient group.

If no majority emerges, the members of the dictator group receive no payoff while the recipients' payoff is drawn from a random distribution between zero and five. ${ }^{1}$

For all treatments, including the nocom treatment, the predictions of standard game theory are straightforward: Purely selfish, profit maximizing agents will individually set the lowest possible transfer of zero and this is the unanimous group decision. Theory assumes rational agents, aware of the rationality of all other players. Therefore, each subject can individually presume to be part of a group featuring homogeneous preferences that acts unanimously. We therefore set our first hypothesis accordingly

Hypothesis 1: All groups will unanimously decide on transfers of

\footnotetext{
${ }^{1}$ Note, that this is not a pure coordination game (see, e.g., Van Huyck et al. 1990, 1991) as the players have individual (unobserved) preferences over the transfer.
} 
$X=0$.

As noted above, this is challenged by models incorporating social preferences which are confirmed by experimental evidence on individual (see Camerer 2003, for an overview), as well as on group dictator games (e.g., Cason and Mui 1994; Luhan et al. 2009). In the framework of group decision-making without any coordination, the assumption of heterogeneous social preferences leads to multiple equilibria. Without any priors on the distribution of social preferences within the group (hence a uniform distribution), the group members will simply set their preferred transfer. Therefore, any transfer out of the action space constitutes an equiprobable majority choice. With the given probability of $7.25 \times 10^{-6}$, however, the occurrence of a majority choice is rather unlikely under this assumption. ${ }^{2}$

According to the model proposed by Fehr and Schmidt (1999), there are two possible equilibria, a "very fair" one at $x=50 \%$ and a "very unfair" one at $x=0 \%$ in the individual dictator game. In our context, these equilibria serve as focal points for possible transfer choices. $^{3}$

Depending on an agent's beliefs about the distribution of "fair" and "selfish" types amongst the group members and her social preferences she will choose either one of these transfers. The reasoning is straightforward: We claim that irrespective of the individual social preferences, each member of the dictator group will choose the transfer she believes to be most likely to be chosen by at least two other group members, as any transfer other than the majority-compatible one will presumably result in a payoff of zero. Even without prior information about the other group members, each of these transfers will lead to a majority choice with a probability of $50 \%$. A selfish agent in a mostly "fair group" will therefore choose a transfer of $50 \%$ as other transfers are irrelevant or will even reduce the probability of a positive payoff. A fair agent in a mostly selfish group will on the other hand only choose a transfer of $50 \%$ if she is willing to sacrifice her payoff of $100 \%$ to gener-

\footnotetext{
${ }^{2} 7.25 \times 10^{-6}$ is the probability to observe three identical numbers when drawing five times from an urn containing 51 numbers and placing the drawn numbers back before each subsequent draw.

${ }^{3}$ Fehr and Schmidt (1999) discuss the possibility of a continuum of fairness equilibria in the dictator game, when abandoning the assumption of piecewise linear inequity aversion. Utility functions that are concave with respect to advantageous inequality result in optimal offers within the range of $[0,0.5]$. In individual decisions subjects with a $\beta>0.5$ will transfer 0.5 , an individual $\beta<0.5$ will lead subjects to transfer 0 . With imperfect information regarding the other group members' preferences it is obvious that the corner solutions - equal to the piecewise linear equilibria of 0 and 0.5 , respectively - will serve as focal points when trying to coordinate on a common transfer.
} 
ate an expected payoff of (the smallest increment more than) $25 \%^{4}$ for the members of the receiver group, which is an unrealistic assumption when assuming the standard parametrization of $0<\alpha \leq \beta \leq 1$ (Fehr and Schmidt, 1999).

When considering social norms, the equal split remains as the only focal point of coordination. The 'Social Comparison Theory' (Festinger, 1954) states that subjects try to present themselves in a way that is socially desirable. Cason and Mui (1994) discussed the implications of this theory in the context of the group dictator game. In absence of any information on the distribution of social preferences the probability of $50 \%$ transfers exceeds the probability of $0 \%$, confirming $50 \%$ as the focal point of coordination. Again, this focal point is found as the modal choice in ultimatum games, where selfish agents strategically choose fair transfers in order to maximize their expected payoffs. Even selfish agents, unaware of the distribution of social preferences within their group, will choose this transfer as it maximizes the probability of a majoritarian choice.

Hypothesis 2: In absence of prior information about the distribution of social preferences within the dictator group, the equal split of $50 \%$ will maximize the probability of a majority choice.

In the star-shaped network, we contend that a focal point is effective. Schelling (1960) describes the impact of any commonly observed information and claims that this information can make any equilibrium a focal point. In our setting, the commonly observed information is not exogenously given, but a part of the actual decision process. In this respect our paper is connected to Eckel and Wilson (2007) who study the impact of "high status" central players in coordination games. Thus in the star treatment, the only equilibrium choice is the proposal of the central member. Not only does her choice constitute $33 \%$ of a possible majority but also this proposal is the only possible point of coordination since it is commonly observed.

Hypothesis 3: In the star-shaped network, the central member's choice is the equilibrium/majority choice.

The central agent, in turn, is not bound to the above-described focal points. Aware of her special position, the central agent can choose her proposal and subsequent transfer according to her individual (social) preferences. Though she observes all proposals from her group, she is bound to stick to her initial proposal because otherwise the coordina-

\footnotetext{
${ }^{4}$ The payoff for the receivers was defined randomly as any amount between 0 and 5 in steps of 0.1. The expected payoff from a random distribution $[0,5]$ in steps of 0.1 equals 2.55 .
} 
tion effect of the commonly observed proposal would be destroyed.

Hypothesis 4: Central members will not be influenced by the other group members' proposals.

We cannot form an a priori hypothesis on whether the star shaped networks will increase or decrease the group transfers as this is subject to the social preferences of the central group members. But we do expect this network structure to significantly increase the number of majority choices in the course of the experiment.

Hypothesis 5: Star-shaped-network groups will reach majority choice more frequently than unconnected groups.

The second network structure we examine is a circular network as depicted in Figure 2. Here the proposals as well as the actual transfers are only observed by the immediate neighbors in the network. Each agent is informed about the proposals and transfers of the agents located to her left and right. This, on the one hand, provides every agent with information about a subgroup large enough to potentially implement a majority choice on their own.

But, on the other hand, both neighbors observe the actions of only one agent out of this subgroup. Therefore even if an agent observes three identical proposals she cannot take this as a secure sign for a majority choice as her neighbors might observe very different proposals and therefore choose an actual transfer differing from the initial proposal. Coordination or compromising on this information subset of three proposals might therefore not be a successful strategy. The extreme case would be that the group members do not take the proposals as valid information and retreat to the focal point of the unconnected groups.

This recourse on the clear focal points would be in line with Elison (1993), and Berninghaus and Schwalbe (1996) who show that local interaction generates risk dominant equilibria. An experimental test is reported in Keser et al. (1998) who embed a three player coordination game in a network structure of eight connected subjects. This circular structure leads subjects to resorted significantly more often (74\%) to the risk dominant strategy than the subjects in the control group $(15 \%)$ despite the entailed lower payoffs. We therefore form two antithetic hypotheses ( 6 and 7 ) for the circular network.

Hypothesis 6: In the circular network, the observed proposals will have a significant impact on the individual transfer choice.

If we apply the same reasoning as in the case of unconnected groups that agents may choose any transfer size with equal probability, the 
chance that a situation occurs in which both received proposals are identical with the one sent is very small. Hence information obtained in a circle is of little value because no agent can trust that others will stick to their proposal. Consequently in the absence of reliable information, the outcome should approach the uninformed and unconnected baseline situation.

Hypothesis 7: In the circular network, the equal split is the equilibrium/majority choice. The observed proposals will have no impact on the individual transfer choice.

\section{Experimental Procedure}

We implemented the above described decision task computerized using z-Tree (Fischbacher 2007) in the Laboratory for Experimental Social and Political Science, University of Oldenburg. Participants were recruited in groups of twenty from a pool consisting of students of all faculties of the University. Four sessions were run for the nocom treatment and six sessions for each the star and the circle treatment, creating eight group observations for the nocom treatment, twelve for the star treatment and eleven for the circle treatment (one session consisted of only ten participants due to low show up).

Upon arrival, the subjects were seated randomly at computer carrels that prevented communicating or seeing the screens of the other players. Instructions were distributed in paper form and a recording of the instructions was played to assure standardized conditions and common information. Subjects were informed about the details of the respective treatment and the communication structure (or the absence of communication).

The experimental currency was 'points' and the official rate of exchange was two Euros per point. So in the lab setup the endowment of the group $\mathrm{C}$ was 25 points and an equal split transfer was $x=2.5$.

We used a one-shot, between-subjects setup with a single decision task with two trials for the formation of a majority. Hence each subject played the game only once. ${ }^{5}$ An experiment lasted ten minutes on average. After the experiment the subjects filled in a short ques-

\footnotetext{
${ }^{5}$ We decided to use a one-shot setup as we are more interested in the pure effect of communication structures than in observing any kind of evolution toward an equilibrium under one of the network structures. With repeated interaction we could observe whether learning patterns are different in different communication networks, which is an interesting question but not the focus of this article. We believe that a one-shot interaction is appropriate for answering our research questions while avoiding the usual problems of repeated interaction, e.g., super game effects.
} 
tionnaire, were paid privately in cash and were released. Subjects that were allocated to the recipient group were asked to fill a questionnaire during the experiment.

\section{Results}

Table 1 summarizes the descriptive statistics from all three treatments. Columns one and two contain the treatments and the number of individual decisions. The third column indicates the number of groups and the fourth presents the number of groups that succeeded in reaching a majority decision in the first or second attempt. Columns five and six contain means and standard deviations of actual group transfers for those groups that attained a majority, columns 7 and 8 contain means and standard deviations for first proposals, that is, the proposals entered in the first stage of the game.

As expected, the decision task was "hardest" in the nocom treatment. Out of the eight groups in the nocom treatment, only three reached a majority choice, one of those only in the second run. These results reinforce our Hypothesis 5: Only $37.5 \%$ of the nocom groups made a majority choice, whereas $83.3 \%$ of the groups accomplished this in the first attempt of the star treatment and still $72.7 \%$ in the first attempt of the circle treatment. Our data does not support Hypothesis 1 while it is congruent with Hypothesis 2 as all three observed group transfers in the nocom treatment were 2.5 points (or 5 Euros).

Table 1: Group transfers and first proposals

\begin{tabular}{lccccccc}
\hline \hline Treatment & $\mathrm{N}$ & Groups & Majority & \multicolumn{2}{c}{ Group transfer } & \multicolumn{2}{c}{ First proposals } \\
\cline { 4 - 7 } & & & & Mean & SD & Mean & SD \\
\hline nocom & 40 & 8 & 3 & 2.5 & 0.00 & - & - \\
star & 60 & 12 & 12 & 1.17 & 1.08 & 1.07 & 0.97 \\
circle & 55 & 11 & 11 & 1.73 & 0.79 & 1.47 & 0.99 \\
\hline \hline
\end{tabular}

Turning to the realized group transfers, we find that they are significantly higher in the nocom treatment than those in the star (MannWhitney-Test $p=0.043)$ and in the circle treatments $(p=0.049)$. However, although the average size of the group transfers is smaller in the star treatment than in the circle treatment, this difference in group transfers between the two network structures is not statistically significant. 
Finally, we compare the initial proposals in the two network structures. With regard to the central subjects in the star networks (member 1) we find that their average proposal (1.26) did not differ significantly $(p=0.635)$ from the proposals of the other group members (1.03). Assuming that the central members were aware of their privileged position, this confirms, on the one hand, that these subjects are not different from the rest of the population. On the other hand, we find that the central network position did not cause behavioral differences in the first place. So we can contend that all the proposals from central group members appear to reflect the true preferences of these agents. The effect of these 'central' proposals is quite striking: Of all 48 remaining subjects, only three proposed the same transfer as the central subject of their respective group, $50 \%$ proposed a higher and $43.7 \%$ a lower transfer than the central agent. In the following decision stage, however, $81.3 \%^{6}$ of all members entered a transfer equal to the proposal of the central member, which is a clear confirmation of Hypothesis 3.

Though the centralized proposal is working as well as theoretically predicted, $50 \%$ of the central subjects did not re-enter the transfer they proposed in the decision stage, leading to a rejection of Hypothesis $4 .{ }^{7}$ Apparently, one half of the central subjects did not realize that their proposal worked as a coordination device. They changed their proposal in the direction of the remaining groups mean proposal, obviously in a mislead attempt to foster coordination.

In the circle treatment we found the proposals entered to be significantly higher (1.47) than those in the star treatment $(1.073 ; p=0.06)$. In the star treatment, the modal proposal of 2.5 was sent by twelve of the 60 subjects, whereas $60 \%$ of the agents chose a proposal of 1.0 or lower. In the circle treatment, $25 \%$ of the subjects sent the modal proposal of 2.5 points and still $20 \%$ proposed a transfer of 2 points. This observed trend towards the equal split in the circular network is in line with Hypothesis 7, though not confirming it.

As stated above, the available information in this network structure is not sufficient to act as a coordination device. The large fraction of 2.5 offers leads us to conclude that subjects realized the inefficiency of information and resort to the focal point of a $50 \%$ offer that would maximize the probability of coordination.

The left panel of Table 2 contains the estimation results for the transfers chosen in the circle treatment conditional on the subjects'

\footnotetext{
${ }^{6}$ This number increases to $87.5 \%$ if we examine only those ten groups of the star treatment which reached a majority in the first attempt.

${ }^{7}$ These inconsistent actions of the central member actually were the reason for both observed failures to form majorities in the first attempt of the star treatment.
} 
own proposal as well as on the respective neighbors' proposals. ${ }^{8}$ We start with the basic linear model reported in the first column of Table 2. We test for heteroskedasticity and omitted variables but can reject both. Using the Ramsey RESET-test for misspecification we rejected the possibility of non-linear combinations of the independent variables. ${ }^{9}$

Unsurprisingly, we find the own proposal (proposal) to have the largest impact on the transfer choice. However, the coefficients of the neighbors' proposals (proposal 1 neighbor) are highly significant and their joint influence on the transfer is even stronger than the own proposal. The large coefficient of the own proposal could be interpreted as partial adherence to the personal preference despite possibly contrary proposals from the neighbors. But this coefficient could also be interpreted as the attempt to equal out the observed diversity of proposals. The high rate of successful majority formations at the first attempt (six out of seven groups) fosters the latter interpretation. This leads us to reject our Hypothesis 7 and to affirm Hypothesis 6 .

Examining other potential factors we do not find a gender effect but a moderate influence of the subjects' age.

In a similar vein, we model the individual transfers in the star treatment as a function of the own proposal, the proposal of the central member as well as age and gender of the subject (right panel of Table 2). As expected, the proposal of the central member (proposal member 1) is decisive for the transfers of all group members, thereby providing further evidence in favor of Hypothesis 3. We find that the best specification is again a linear regression with only the own proposal and the proposal of the central agent as explanatory variables (column 6 of Table 2). Still, the influence of the own proposal is only a minor one and might as well be neglected, as displayed by the BICstatistics.

\section{Conclusion}

In this paper we studied the impact of different communication network structures on group decision making. The decision task was

\footnotetext{
${ }^{8}$ We found no significant difference of first or second trials to form a majority. This might be due to the low numbers of observations for second trial majorities. We therefore excluded the "trial" variable from the estimation reported in Table 2.

${ }^{9}$ We tested logarithmic transformation of both dependent and independent variables, which did not improve the quality of the estimation.
} 
Table 2: Estimation results for both network types

\begin{tabular}{lcc|ccccc}
\hline \hline Dependent Variable: transfer \\
\hline \multicolumn{2}{c}{ Circle } & \multicolumn{7}{c}{ Star } \\
\hline Network & $0.452^{* * *}$ & $0.455^{* * *}$ & $0.226^{* * *}$ & $0.223^{* *}$ & $0.200^{* *}$ & $0.200^{* *}$ & \\
proposal & $(7.33)$ & $(7.51)$ & $(2.52)$ & $(2.57)$ & $(2.55)$ & $(2.58)$ & \\
& 0.044 & & -0.033 & & 0.005 & & \\
age & $(0.36)$ & & $(0.18)$ & & $(0.03)$ & & \\
& 0.012 & 0.013 & -0.011 & -0.01 & & & \\
proposal 1 & $(1.30)$ & $(1.36)$ & $(1.28)$ & $(1.27)$ & & & \\
neighbor1 & $0.393^{* * *}$ & $0.390^{* * *}$ & & & & & \\
proposal 1 & $(6.56)$ & $(6.62)$ & & & & & \\
neighbor2 & $0.353^{* * *}$ & $0.357^{* * *}$ & & & & & \\
proposal & $(5.88)$ & $(6.15)$ & & & & & \\
member 1 & & & $0.765^{* * *}$ & $0.769^{* * *}$ & $0.785^{* * *}$ & $0.785^{* * *}$ & $0.772^{* * *}$ \\
constant & $-0.657^{* *}$ & $-0.644^{* *}$ & $(9.29)$ & $(9.95)$ & $(10.33)$ & $(10.86)$ & $(10.17)$ \\
\multicolumn{1}{c|c}{} & $(2.36)$ & $(2.36)$ & $(0.67)$ & 0.127 & -0.126 & -0.122 & 0.1 \\
\hline observations & 55 & 55 & 48 & 48 & $(0.79)$ & $(1.26)$ & $(1.63)$ \\
AIC & 1.17 & 1.14 & 1.78 & 1.74 & 1.76 & 1.71 & 1.78 \\
BIC & -144.03 & -147.89 & -90.89 & -94.72 & -94.11 & -97.98 & -96.44 \\
Adjusted $R^{2}$ & 0.79 & 0.79 & 0.69 & 0.7 & 0.69 & 0.7 & 0.67 \\
\hline \hline
\end{tabular}

Robust t-statistics in parentheses

${ }^{*}$ significant at $10 \%$; ${ }^{* *}$ significant at $5 \%$; ${ }^{* *}$ significant at $1 \%$

modeled as a group dictator game as in our view this reflects the intra group conflicts that a heterogeneous committee might face as well as the motivational structure of preferences and responsibilities for a third party that is at the core of political decision making in the problem-solving mode.

We compared unconnected groups that had no possibility to communicate for coordination with a star-shaped network with one central, commonly observed member and a circular network where communication was possible only with the immediate neighbors.

As expected, both network types increased the rate of successful decision making significantly. Both network structures produced a $100 \%$ success rate of majoritarian decisions. In the star treatment, the group decisions are solely driven by the central agent. Whatever proposal this agent makes is subsequently adopted as a group decision. Groups even formed a majority in those occasions where the central agent changed the proposed transfer amount between the proposal 
phase and the decision phase. ${ }^{10}$ We thus find strong evidence for the power attributed to the central agent if information about the intention of other members is provided selectively. Furthermore, although the whole range of possible transfers has been chosen, on average these central agents select rather low transfer shares.

The information provided for coordination in the circular network is less efficient and correctly evaluated as such by the subjects. The proposals made get closer to the equal split, which is also selected by successful groups without any information. This trend towards the socially accepted transfer of $50 \%$ of the pie - and therefore towards the risk dominant strategy - appears to be the reason for the high rate of successful coordination attempts and majority decisions in the circular treatment. This is in line with the findings of Keser et al. (1998) and Ellman and Pezanis-Christou (2010). When actually in possession of the information, however, subjects do use this information to attempt to coordinate within this subgroup.

Our results show a clear impact of the structure of communication networks on the final outcome of group decision making. Our scope is of course a very narrow one, comparing only three types of networks. Further research could examine other network features such as the size of the decision making group, or changing network structures. Moreover, different learning patterns in the different network structures might either result in convergence or further differentiation of outcomes. Another promising avenue for research is to explore the extent to which variation in the communication content, such as the possibility to provide arguments in favor of a proposal, affects the outcome.

\footnotetext{
${ }^{10}$ This result is puzzling because changing the proposed transfer is not a rational strategy for the central player. The only ad hoc explanation we have, in view of a large number of freshmen in the pool not previously exposed to the kind of reasoning necessary for the task, is that half of the central players did not sufficiently understand their power and responsibility. But this puzzle remains for further exploration.
} 
Bala V, Goyal S (2000) A noncooperative model of network formation. Econometrica 68:1181-1229.

Beyres J (2004) Voice and access. Political practices of European interest associations. European Union Politics 5:211-40.

Biliouri D (1999) Environmental NGOs in Brussels: How powerful are their lobbying activities? Environmental Politics 8:173-82.

Blount S (1995) When social outcomes aren't fair: The effect of causal attributions on preferences. Organizational Behavior and Human Decision Processes 63:131-144.

Bolton G, Ockenfels A (2000) ERC: A theory of equity, reciprocity, and competition, American Economic Review 90:166-193.

Bouwen P (2004) Exchanging access goods for access: A comparative study of business lobbying in the European Union institutions. European Journal of Political Research 43:337-69.

Brosig J, Weimann J, Ockenfels A (2003) The effect of communication media on cooperation. German Economic Review 4:217-241.

Camerer C (2003) Behavioral game theory: Experiments in strategic interaction, Princeton University Press, Princeton.

Camerer C, Thaler RH (1995) Anomalies: Ultimatums, dictators and manners. Journal of Economic Perspectives 9:209-219.

Cason T, Mui V-L (1997) A laboratory study of group polarization in the team dictator game. Economic Journal 107:1465-1483.

Charness G, Corominas-Bosch M, Fréchette G (2007) Bargaining and network structure: An experiment. Journal of Economic Theory 136,2865.

Chwe M (2000) Communication and coordination in social networks, Review of Economic Studies 67, 1-16.

Crombez C (2002) Information, lobbying and the legislative process in the European Union. European Union Politics 3:7-32.

Dutta B, Jackson M (2003) On the formation of networks and groups, 
in: Dutta B, Jackson M (Eds.),Networks and Groups: Models of Strategic Formation, Springer, Berlin.

Ellman M, Pezanis-Christou P (2010) Organizational Structure, Communication, and Group Ethics. American Economic Review.

Eckel CC, Wilson RK (2007) Social Learning in Coordination Games: Does Status Matter? Experimental Economics 10, 317-329.

Eising R (2007) Institutional context, organizational resources and strategic choices. Explaining interest group access in the European Union. European Union Politics 8:329-62.

Festinger L (1954) A theory of social comparison processes. Human Relations 7:117-140.

Fehr E, Schmidt KM (1999) A theory of fairness, competition, and cooperation, Quarterly Journal of Economics 114:817-868.

Frohlich N, Oppenheimer JA (2007) Justice preferences and the Arrow problem, Journal of Theoretical Politics 19: 363-390.

Glaeser E, Sacerdote B, Sheinkman S (1996) Crime and social interactions, Quarterly Journal of Economics 111, 507-548.

Goyal S (2007) Connections: An introduction to the economics of networks, Princeton University Press, Princeton.

Greenwood J (2003) Interest representation in the European Union. Palgrave, Basingstoke.

Grossman GM, Helpman E (2001): Special interest politics. MIT Press, Cambridge.

Hallstrom, LK (2004) Eurocratising enlargement? EU elites and NGO participation in European environmental policy. Environmental Politics 13:175-93.

Jackson,M, Wolinsky, A (1996) A strategic model of social and economic networks, Journal of Economic Theory 71, 44.

Jackson M, Watts A (1998) The Evolution of Social and Economic Networks, Caltech WP, 1044. 
Keser C, Erhart K-M, Berninghaus SK (1998) Coordination and local interaction: experimental evidence. Economics Letters 58: 269-275.

Kahneman D, Knetsch J, Thaler R (1986) Fairness as a constraint on profit seeking: Entitlements in the market. American Economic Review 76:728-741.

Kosfeld, M (2004) Economic Networks in the Laboratory: A Survey. Review of Network Economics 3: 20-42.

Luhan WJ, Kocher MG, Sutter M (2009) Group polarization in the team dictator game reconsidered. Experimental Economics 12:26-41.

Mahoney C (2004) The power of institutions. State and interest group activity in the European Union. European Union Politics 5:441-66.

Morris S (2000) Contagion, Review of Economic Studies 67, 67-78.

Ochs J (1995) Coordination problems. In: Kagel J, Roth AE (ed) Handbook of experimental economics. Princeton University Press, Princeton, pp 195-251.

Pillutla MM, Murnighan JK (1995) Being fair or appearing fair: Strategic behavior in ultimatum bargaining. Academy of Management Journal 38:1408-1426.

Potters J, Sloof R (1996) Interest groups: A survey of empirical models that try to assess their influence. European Journal of Political Economy 12:403-42.

Roth AE (1995) Introduction to experimental economics. In: Kagel J, Roth AE (ed) Handbook of experimental economics. Princeton University Press, Princeton, pp 3-109.

Scharpf FW (1997) Games real actors play. Actor-centered institutionalism in policy research. Westview Press, Boulder Schelling T (1960) The strategy of conflict. Harvard University Press, Cambridge.

Sia CL, Tan BCY, Wei KK (2002) Group polarization and computermediated communication: Effects of communication cues, social presence, and anonymity. Information Systems Research, 13:70-90. 
Thomson R, Stokman F, Achen CA, König T (ed) (2006) The European Union decides. Cambridge University Press, Cambridge.

Traub S, Seidl C, Schmidt U (2009) An Experimental Study on Individual Choice, Social Welfare, and Social Preferences. European Economic Review 53: 385-400.

Tyran JR, Sausgruber R (2006) A Little Fairness May Induce a Lot of Redistribution in Democracy. European Economic Review 50: 469385 .

Wellman B, Berkowitz S (1988) Social Structure: A Network Approach. Cambridge University Press,Cambridge.

Van Huyck JB, Battalio RC, Beil RO (1990) Tacit coordination games, strategic uncertainty, and coordination failure. American Economic Review 80:234-48.

Van Huyck JB, Battalio RC, Beil RO (1991) Strategic uncertainty, equilibrium selection, and coordination failure in average opinion games. The Quarterly Journal of Economics 106: 885-910. 


\title{
Appendix: Experimental Instructions 'star' treatment
}

\author{
Welcome to the experiment and thank you for your \\ participation! \\ Please do not talk to other participants until the end of the \\ experiment!
}

\section{Instructions}

The aim of this experiment is the investigation of decision behaviour. During the experiment, you and the other participants will be asked to make decisions. You will earn money in doing so. The amount of your payoff is determined by your own decisions and by those made by other participants.

\section{2 types of participants}

There are two types of participant: type A and type B. You will be randomly assigned to one of these types/roles. Your type/role will be displayed at the upper border of the screen during the experiment.

\section{Duration}

The entire experiment will last for approx. 45 minutes. If you have any questions after reading the instructions, please raise your hand. One of the experimenters will come to you and answer your questions privately.

\section{Payoffs}

Your earnings depend on your decisions and the decisions of other participants in this experiment (see below). You will be paid to you privately and in cash at the end of the experiment.

\section{Anonymity}

The identities of the participants that have interacted with you will never be revealed to you. The other participants will not learn either during or after the experiment which role you were playing and how much you have earned.

\section{Grouping}

You will be teamed up with four other participants of the same type. Your position within the group (member 1-5) will be randomly determined and displayed on the screen. Each group of type A is randomly matched with one group of type B. 


\section{Initial endowment}

Each member of group A receives 5 points as initial endowment.

One point is worth 2 EURO, so your initial endowment corresponds to 10 EURO.

The members of group B do not receive an initial endowment.

\section{Decision group A: Choice of a transfer}

Each Participant of group A individually specifies a transfer that determines which part of his/her initial endowment will be transferred to group B. Each amount between 0 and 5 points (in steps of 0.1 ) can be selected. It is possible to transfer nothing or the whole 5 points.

If the members group A choose $\mathrm{x}$ points, this means that this amount $\mathrm{x}$ will be transferred from each member of group A to one member of group B.

Prior to the transfer choice the members of group A have to post a proposal for this transfer. These individual proposals will be displayed to specific members of the team as displayed in figure 1 .

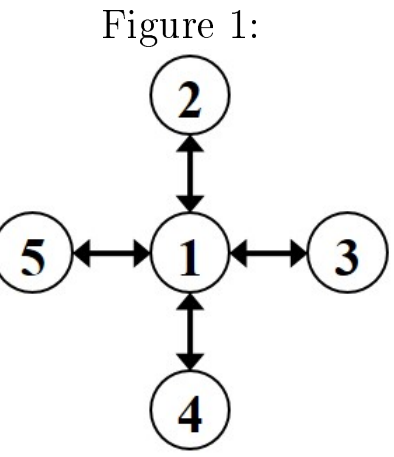

Player 1 will be displayed all proposals from his teammates. All other team members will see only the proposal from member 1 .

After all proposals were displayed the members select the transfer individually.

This concludes stage 1 for participant $A$.

Majority: If more than half of all group members (3) have selected the same transfer this constitutes a majority decision. 
If no majority decision is found group A may attempt to do so in phase 2. The group members can again make individual proposals and select a transfer in this second phase.

Group B cannot take any action. The members of group B will be asked to fill in a questionnaire individually.

\section{Results/earnings}

Alternative 1.: A majority decision was found. The transfer chosen by the majority of group $\mathrm{A}$ is transferred to group B.

Earnings of each member of group $A=5$ points - Transfer

Earnings of each member of group B $=$ Transfer

Alternative 2.: No majority decision was found.: If there is no majoritarian choice of a transfer in group a after phase 2, the members of group A do not receive any payoff.

The members of group B receive a random draw from the distribution of $[0,2.5]$ points as a payoff.

\section{End}

After filling in a short questionnaire you will be displayed your total earnings in Euro. You will be paid your total earnings privately and in cash. 